\title{
INFRARED SPECTROSCOPY OF NEARBY RADIO ACTIVE ELLIPTICAL GALAXIES
}

\author{
Jeremy Mould ${ }^{1,2,9}$, Tristan Reynolds ${ }^{3}$, Tony Readhead ${ }^{4}$, David Floyd ${ }^{5}$, Buell Jannuzi ${ }^{6}$, Garret Cotter ${ }^{7}$, \\ Laura Ferrarese $^{8}$, Keith Matthews ${ }^{4}$, David Atlee ${ }^{6}$, and Michael Brown ${ }^{5}$ \\ ${ }^{1}$ Centre for Astrophysics and Supercomputing Swinburne University, Hawthorn, Vic 3122, Australia; jmould@ @ swin.edu.au \\ ${ }^{2}$ ARC Centre of Excellence for All-sky Astrophysics (CAASTRO) \\ ${ }^{3}$ School of Physics, University of Melbourne, Melbourne, Vic 3100, Australia \\ ${ }^{4}$ Palomar Observatory, California Institute of Technology 249-17, Pasadena, CA 91125 \\ ${ }^{5}$ School of Physics, Monash University, Clayton, Vic 3800, Australia \\ ${ }^{6}$ Steward Observatory, University of Arizona (formerly at NOAO), Tucson, AZ 85719 \\ ${ }^{7}$ Department of Physics, University of Oxford, Denys, Oxford, Keble Road, OX13RH, UK \\ ${ }^{8}$ Herzberg Institute of Astrophysics Herzberg, Saanich Road, Victoria V8X4M6, Canada \\ Received 2012 June 6; accepted 2012 September 26; published 2012 November 1
}

\begin{abstract}
In preparation for a study of their circumnuclear gas we have surveyed $60 \%$ of a complete sample of elliptical galaxies within $75 \mathrm{Mpc}$ that are radio sources. Some $20 \%$ of our nuclear spectra have infrared emission lines, mostly Paschen lines, Brackett $\gamma$, and [Fe II]. We consider the influence of radio power and black hole mass in relation to the spectra. Access to the spectra is provided here as a community resource.
\end{abstract}

Key words: galaxies: elliptical and lenticular, $\mathrm{cD}$ - galaxies: nuclei - infrared: general - radio continuum: galaxies

\section{INTRODUCTION}

Astrophysicists have striven to learn the detailed physics of active galactic nuclei (AGNs) since black holes (BHs) were first posited as the energy source (Lynden-Bell \& Rees 1971). Landmarks were the dusty torus model (Mayes et al. 1985) and the unified scheme of AGNs, which realized the implications of this geometry (Antonucci et al. 1987) from the observer's standpoint. With the advent of laser guide star adaptive optics on $10 \mathrm{~m}$ class telescopes, the opportunity now exists to image the circumnuclear gas in nearby active galaxies and, with the aid of detailed modeling, to understand the physics of the central $10-201 \mathrm{t}-\mathrm{yr}$ of these powerful engines. The purpose of the project our small team has recently commenced is to study a complete sample of galaxies that are radio sources within $40 \mathrm{Mpc}$. Our work focuses first on the elliptical galaxies, as these are the simplest in terms of stellar population and gas and dust content. We emphasize nearby galaxies, as at $20 \mathrm{Mpc}$ the diffraction-limited resolution of the OSIRIS instrument of the Keck Observatory is $3.2 \mathrm{pc}$, which is appropriate for disk gas heated by AGNs. ${ }^{10}$ Central BHs are now believed to be basic constituents of most, if not all, massive galaxies (Magorrian et al. 1998; Kormendy 2004). That BH growth is linked with galaxy formation was realized because of the correlation of $\mathrm{BH}$ mass with the properties of the host galaxy (Kormendy 1993; Kormendy \& Richstone 1995; Magorrian et al. 1998; Gebhardt et al. 2000; Ferrarese \& Merritt 2000; Barth et al. 2005; Greene \& Ho 2006), although alternative explanations have been put forth (Jahnke \& Maccio 2010).

The deviation of the galaxy mass function from the strict power law describing dark matter halo masses provides an additional and independent argument in favor of feedback (e.g., Hopkins et al. 2008). The feedback is also relevant to the cooling flow problem, particularly with respect to radio AGNs (e.g., Bower et al. 2006; Best et al. 2006). Studies have shown that

\footnotetext{
9 Visiting Astronomer, Kitt Peak National Observatory, National Optical Astronomy Observatory, which is operated by the Association of Universities for Research in Astronomy (AURA) under cooperative agreement with the National Science Foundation.

${ }^{10}$ Our sample mean is actually $40 \mathrm{Mpc}$, where the OSIRIS resolution is $6.4 \mathrm{pc}$.
}

$\sim 30 \%$ of the most massive galaxies are radio continuum sources (e.g., Fabbiano et al. 1989; Sadler et al. 1989). As a direct manifestation of accretion, and therefore BH growth, AGNs and the consequences of their energy feedback have figured prominently in most current ideas of structure formation (e.g., Granato et al. 2004; Springel et al. 2005; Hopkins \& Hernquist 2006). Awareness of the importance of BHs has engendered broad interest in the study of the AGN phenomenon itself.

This paper focuses on nuclear activity in nearby galaxies. By selection, most of the objects occupy the faintest end of the AGN luminosity function and have low-accretion rates. Although energetically less impressive, low-luminosity AGNs deserve scrutiny. By virtue of proposed reasons such as duty cycle (Greene \& Ho 2007) or high/low-accretion states (see, e.g., Hardcastle et al. 2006), most AGNs are found in a lowluminosity state. Brown et al. (2011) argue that all massive early-type galaxies are radio sources, mostly powered by AGNs (Slee et al. 1994; Ho 1999). The advent of new telescopes and new analysis techniques yield fresh insights into this problem.

The aims of our project are (1) to image nearby ellipticals that are radio sources in 1-2 $\mu \mathrm{m}$ emission lines, (2) to deduce the kinematics of the gas in these lines, (3) to model the density and temperature of the gas, (4) to deduce the mass of the nuclear $\mathrm{BH}$ and the star formation rate (SFR) in the central $10 \mathrm{pc},(5)$ to measure gas inflow and outflow rates in non-equilibrium regions of the central structure, and (6) to summarize these results over the $40 \mathrm{Mpc}$ complete sample of ellipticals, noting correlations with mass, radio power, SFR, emission-line strength, and X-ray power.

In this paper, we present the first half of a survey of infrared emission lines carried out at Palomar Observatory and Kitt Peak National Observatory. A number of the galaxies show lines of [Fe II], which will be followed up with $8 \mathrm{~m}$ class telescopes to accomplish the first goal of our project. Circumnuclear SFRs are presented for those with Brackett $\gamma$ in emission.

\section{OBSERVATIONS}

Brown et al. (2011) have compiled a complete sample to $K=9$ of 396 elliptical galaxies with declination $>-40^{\circ}$. The 

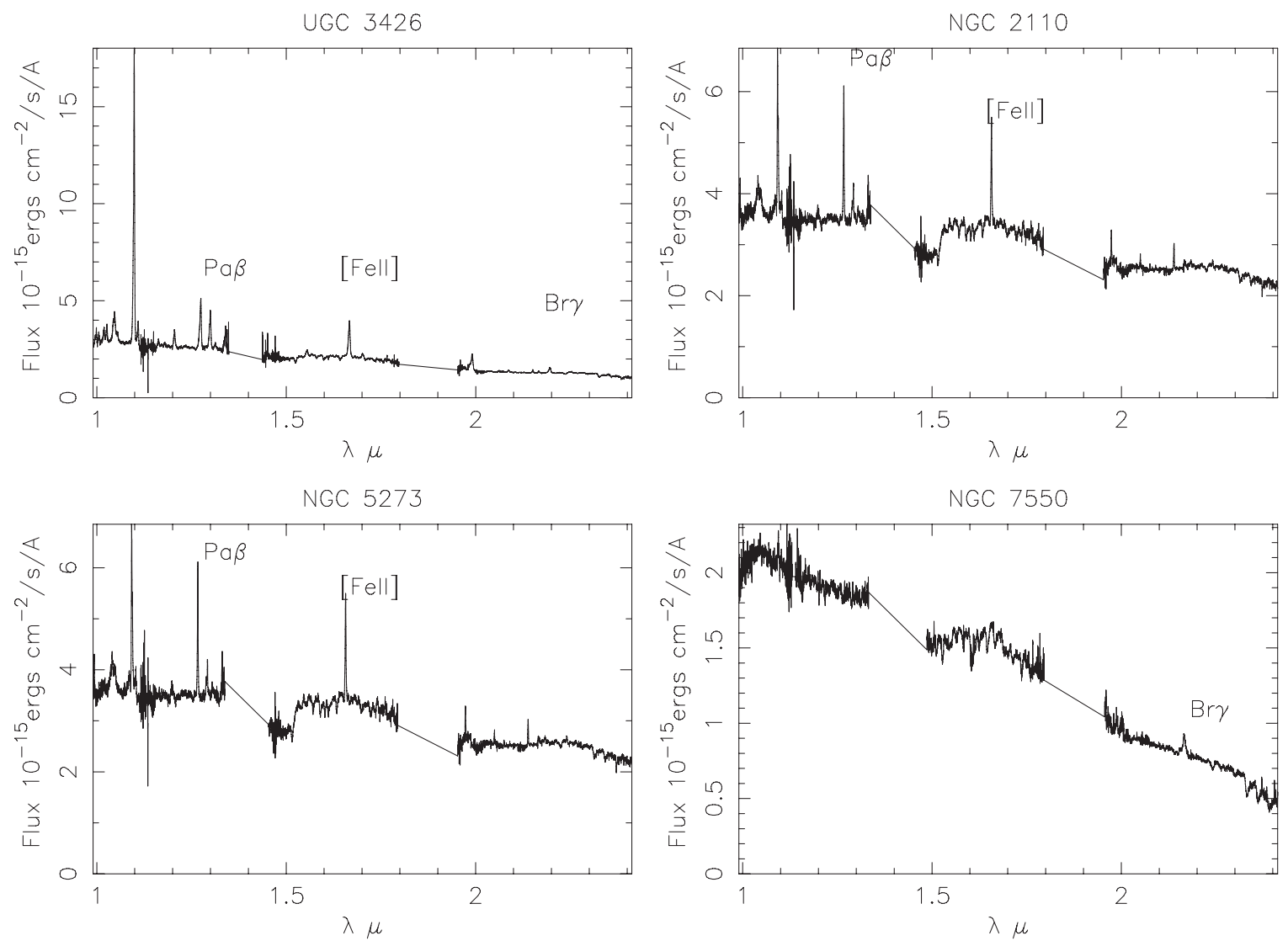

Figure 1. Palomar Triplespec spectra ( $1^{\prime \prime}$ aperture) of three of the strongest emission-line galaxies encountered in the sample so far. [Fe II] is the strong line at $1.64 \mu \mathrm{m}$. $\mathrm{P} \beta$ is at 1.28 and $\mathrm{Br} \gamma$ at $2.18 \mu \mathrm{m}$. NGC 7550 has $\mathrm{Br} \gamma$ in emission, indicating a high star formation rate.

\begin{tabular}{lcccccc}
\multicolumn{7}{c}{$\begin{array}{c}\text { Table 1 } \\
\text { Runs }\end{array}$} \\
\hline \hline No. & Telescope & Spectrograph & \multicolumn{1}{c}{ Dates } & Resolution & Slit & Wavelength Range \\
\hline 1 & Hale 5 m Palomar & Triplespec & 2011 Sep 14-15 & 2600 & $1^{\prime \prime} 4$ pixels & $1.0-2.4 \mu \mathrm{m}$ \\
2 & Mayall 4 m KPNO & Flamingos & 2011 Nov 6-11 & 1000 & $1^{\prime \prime}$ 3 pixels & $0.9-1.8 \mu \mathrm{m}$ \\
3 & Hale 5 m Palomar & Triplespec & 2012 Jan 2 & 2600 & $1^{\prime \prime}$ & $1.0-2.4 \mu \mathrm{m}$ \\
4 & Mayall 4 m KPNO & Flamingos & 2012 Feb 4-9 & 1000 & $1^{\prime \prime}$ & $0.9-1.8 \mu \mathrm{m}$ \\
5 & Hale 5 m Palomar & Triplespec & 2012 May 29-30 & 2600 & $1^{\prime \prime}$ & $1.0-2.4 \mu \mathrm{m}$ \\
\hline
\end{tabular}

Notes. The following radio active early-type galaxies have Palomar Triplespec spectra from J. Woo et al. (2012, in preparation): NGC 3245, 3607, 3608, 4261, 4291, 4374, 4459, 4473, 4486, 4564, 4596, 4649, 4697, 4742, 6251, and 7052. None of these galaxies have emission lines in the $H$ band. The Triplespec detector format is $2048 \times 1024$; the Flamingos detector is $2048^{2}$ pixels.

231 detected radio sources ${ }^{11}$ among them are the basic sample for this paper. For diffraction-limited imaging of the centers of these galaxies with the Keck and Gemini Observatories, we have begun to observe the subsample with $1.4 \mathrm{GHz}$ radio power $>10^{21} \mathrm{~W} \mathrm{~Hz}^{-1}$ and declination $>-36^{\circ}$ with the instrumentation listed in Table 1. Sample near-infrared spectra are shown in Figures 1-5 in which no redshift correction has been applied. Currently, $20 \%$ of the galaxies observed are showing emission lines of [Fe II] and/or $\mathrm{Br} \gamma$, suitable for follow-up with Keck OSIRIS and Gemini North NIFS integral field unit (IFU)

${ }^{11}$ Galaxies for which upper limits were given in Brown's data table were omitted. spectrographs. ${ }^{12}$ Our five runs (Table 1) with infrared slit spectrographs have netted nuclear spectra of 136 galaxies from the basic sample. This is sufficient to understand the statistics of circumnuclear properties in current epoch ellipticals. Areas of notable incompleteness are the greater Virgo cluster area and the southern hemisphere. Black hole masses in Table 4 are based on the $K$-band magnitude, following Graham (2007).

At both telescopes all galaxies were observed in two slit positions, A and B, separated by $20^{\prime \prime}$, in an ABBA sequence of 4-5 minute exposures. Following the object sequence, an eighth magnitude A0V star was observed within an air mass of 0.1 of the object for telluric correction. This was also used for spectrophotometric calibration, which employed Vega model

\footnotetext{
12 For all but the faintest nuclei, on-axis guiding is possible.
} 

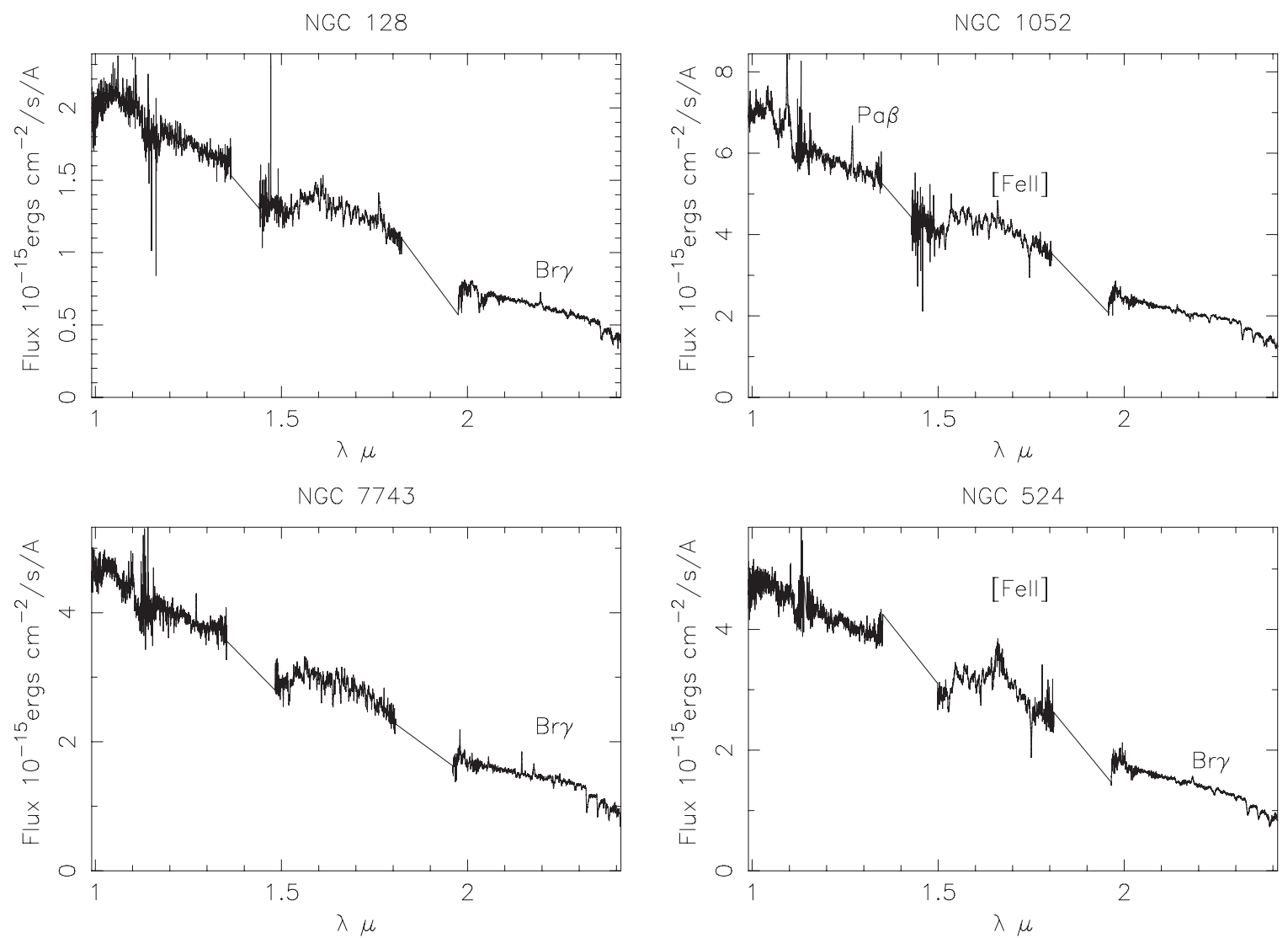

Figure 2. Palomar Triplespec spectra of emission-line nuclei. The absorption features beyond $2.3 \mu$ are CO band heads from the stellar nucleus.

fluxes for zeroth visual magnitude. For wavelength calibration an argon lamp was used at Kitt Peak and telluric $\mathrm{OH}$ lines were used at Palomar.

The galaxies with emission lines in these near-infrared spectra are listed in Table 2; those without are in Table 3, which also gives the signal-to-noise ratio of the Kitt Peak spectra at $1.01 \mu$ and Palomar spectra at $1.04 \mu$.

\section{DATA REDUCTION}

The data sets from each observatory required different data reduction packages. The Kitt Peak National Observatory data was reduced using IRAF and the reduction of the Palomar Observatory data was done using Spextool v4.0 beta. (Cushing et al. 2004).

The first step in the reduction of the Kitt Peak data was the creation of the flat field and dark images. The flat field was created by subtracting the flat lamps-off image from the flat lamps-on image. The first and second nights' data had separate flat-field images. The third night's data set did not contain flatfield images, so the flat-field image created for night 2 was also used for night 3 objects.

For the standard stars, from the observation files the dark image was subtracted and then the flat-field image was divided out. The spectrum was then extracted using the task "apall" in IRAF. As the observation files for the galaxies were taken as ABBA, the galaxy spectra were extracted by creating images for $\mathrm{A}-\mathrm{B}$ and $\mathrm{B}-\mathrm{A}$ files, which were then divided by the flat-field image. The flat fielded $\mathrm{A}-\mathrm{B}$ and $\mathrm{B}-\mathrm{A}$ images were then rotated and cropped before running "apall" on each image.

The axes for the spectra were initially in terms of pixels and had to be wavelength calibrated using an argon arc spectrum.
This was achieved by picking five prominent peaks in the arc spectrum and identifying the pixel value corresponding to the wavelength of each peak. These five values were then used to interpolate along the entire spectrum.

To create the flat-field image used with the Palomar data, IRAF was first used to form a set of flat-field images by subtracting the individual flat lamp-off images from the flat lamp-on images. The flat-field images created with IRAF were then used in the program "xspextool" in Spextool to create the master flat field. The wavelength calibration was created in "xspextool" using any four galaxy images. The spectra for the galaxies and standard stars were extracted in "xspextool" for $\mathrm{A}-\mathrm{B}$ and $\mathrm{B}-\mathrm{A}$ image sets using the master flat-field image and the wavelength calibration.

The individual extracted spectra for each galaxy and standard star were then combined using "xcombspec" by selecting all images of each object and scaling all orders with respect to the spectrum with the greatest magnitude in the order 3 spectrum.

Telluric correction was carried out on the combined spectra for each galaxy using the combined standard star spectra with a similar air mass to the galaxy using the Spextool program "xtellcor". The hydrogen absorption lines in the standard star spectrum were rescaled for all orders and the telluric spectrum was constructed. The four orders $(3,4,5,6)$ of the telluriccorrected galaxy spectra were merged using "xmergeorders" to give a single spectrum.

Spectra are provided for readers desiring access to them. ${ }^{13}$ Cesetti et al. (2009) show spectra of NGC 4649, NGC 4697,

\footnotetext{
13 https://sites.google.com/site/radioactivegalaxies/
} 


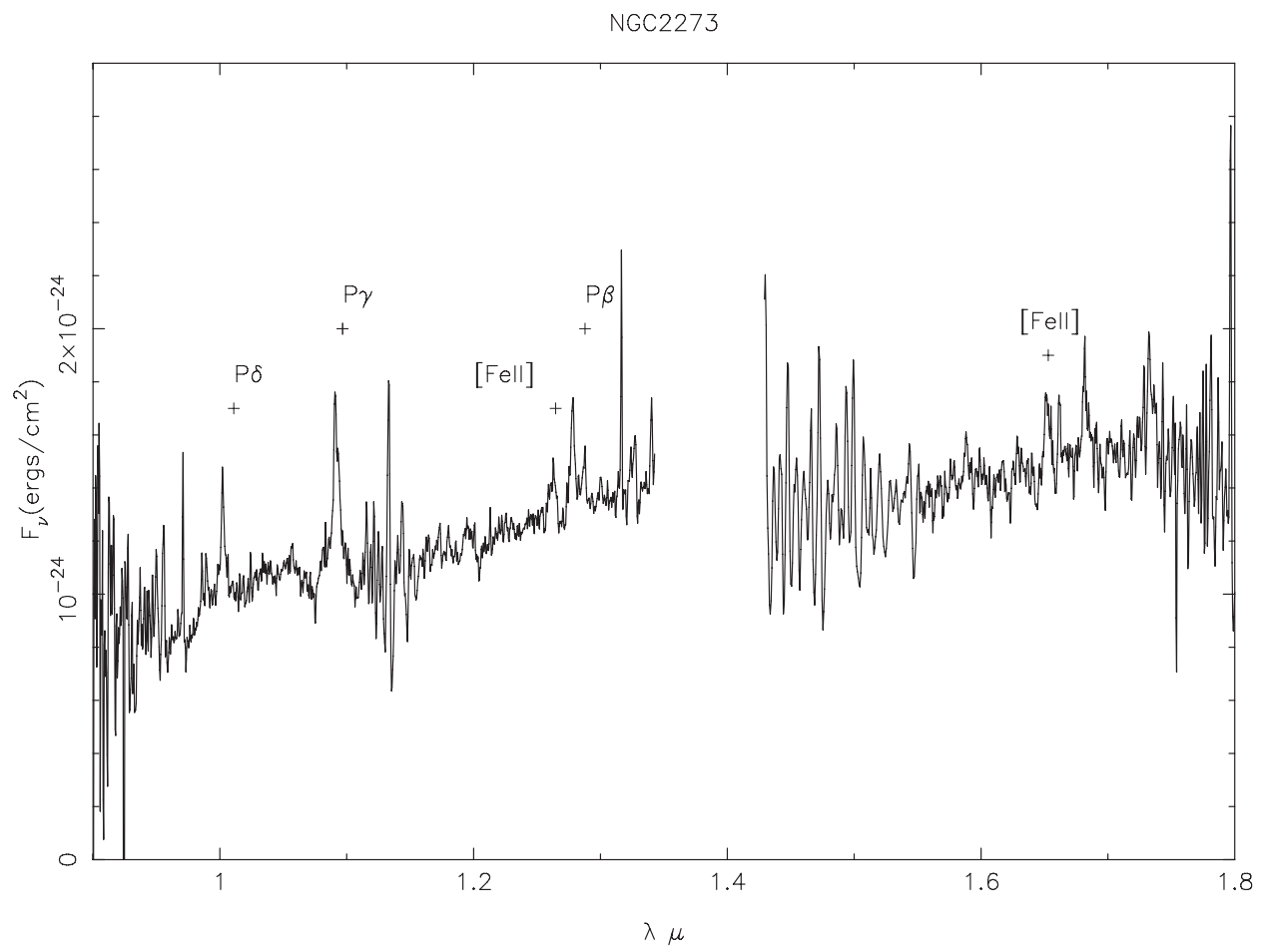

Figure 3. Flamingos spectrum of NGC 2273. The Paschen emission lines, such as $\mathrm{P} \gamma 1.09 \mu$, may thus be exaggerated. Three-point boxcar smoothing has been applied.
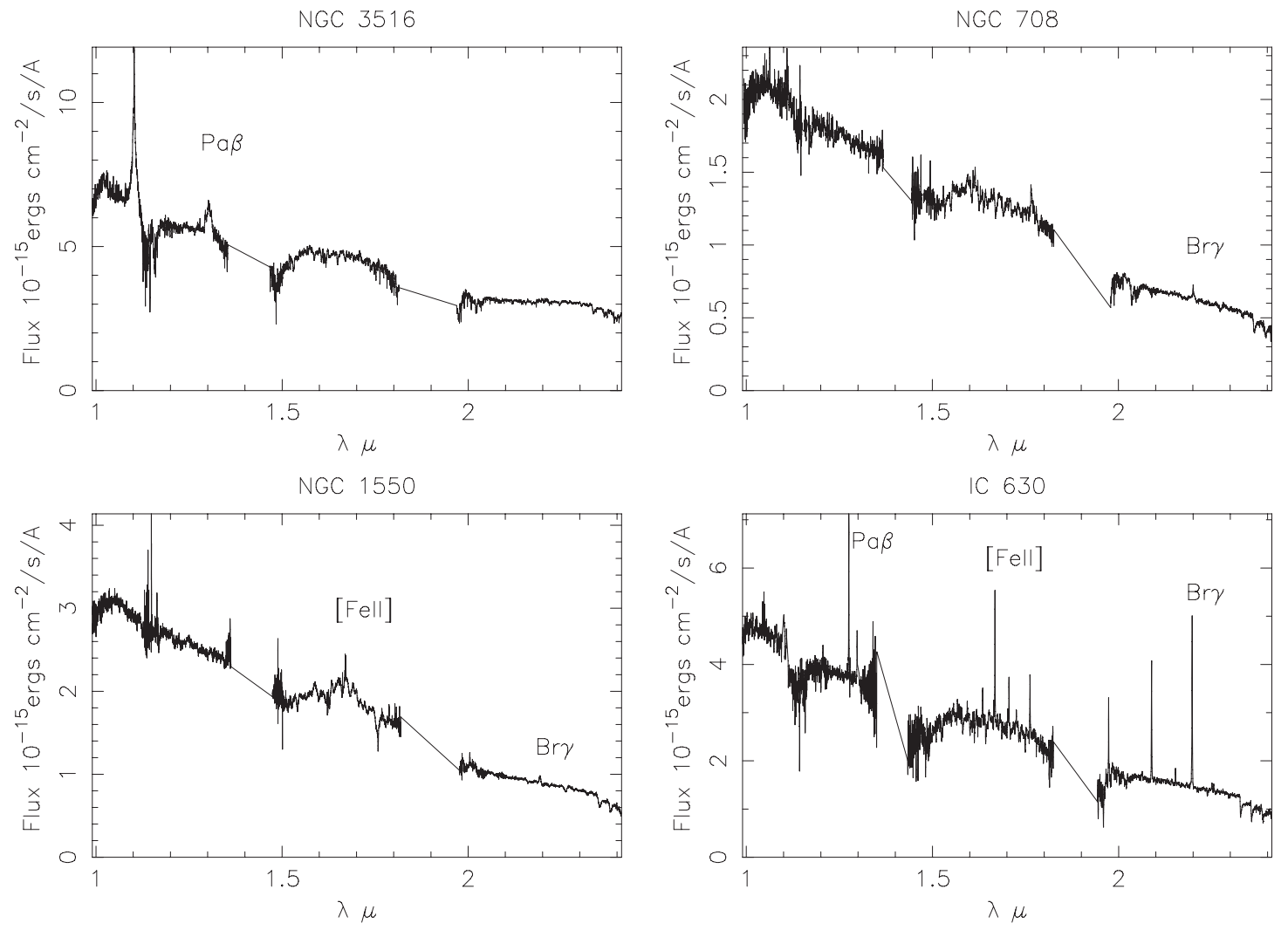

Figure 4. Further Palomar spectra of emission-line nuclei.

IC 4296, NGC 4564, NGC 5077, and NGC 5576. Riffel et al. (2006) show a spectrum of NGC 2110.

\section{DISCUSSION}

We compare the radio power of our emission-line galaxies with that of the full 397 galaxy Brown et al. sample in Figure 6.
Infrared emission-line nuclei have six times stronger radio emission than average at a given $K$-band magnitude (or stellar mass). This does not stand out in the figure, but is a $4 \sigma$ effect, based on the line in Figure 6 and the scatter in the relation. Both radio and infrared quantities here are from the data table of Brown et al. The whole-sample correlation may be expected on general scaling grounds. A larger galaxy hosts a larger $\mathrm{BH}$, 
IC 459
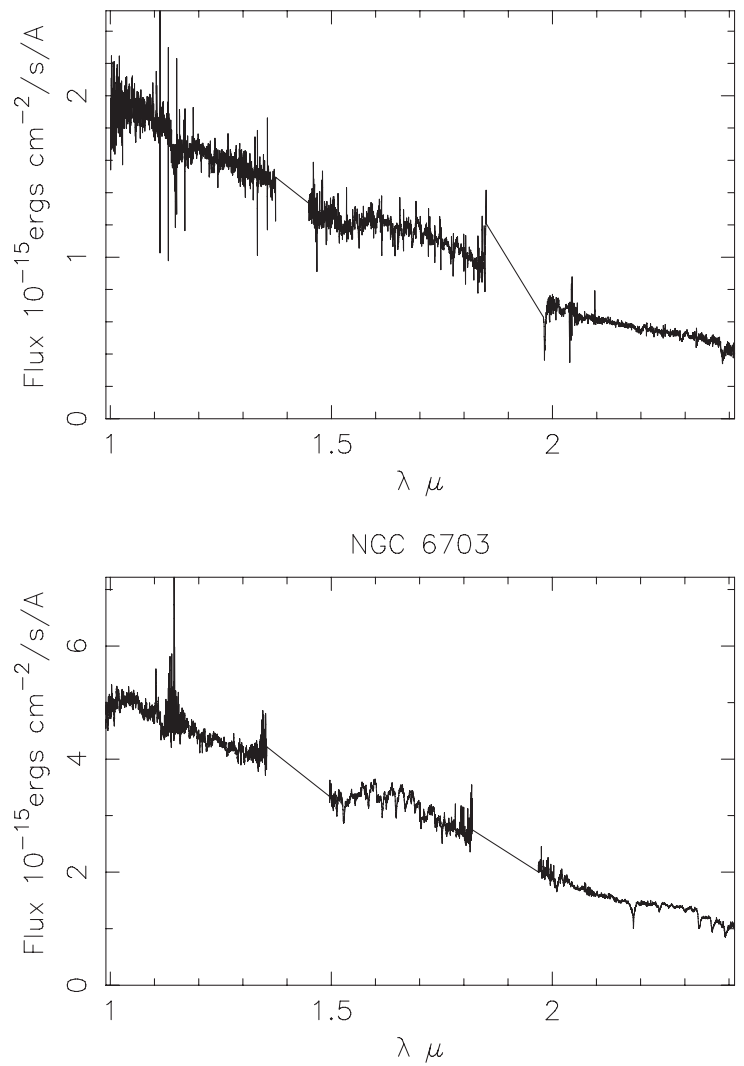

NGC 6251
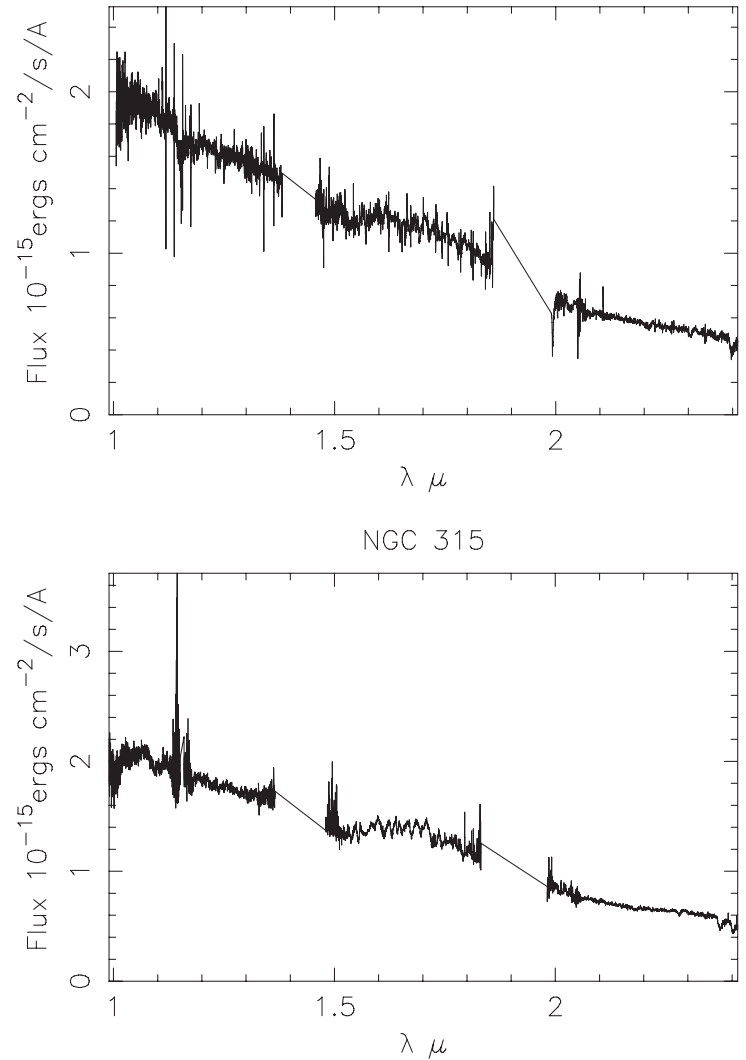

Figure 5. Palomar spectra of absorption-line nuclei. CO band heads are seen at the red end of the $2 \mu$ window. NG 6703 has Br $\gamma$ in absorption.

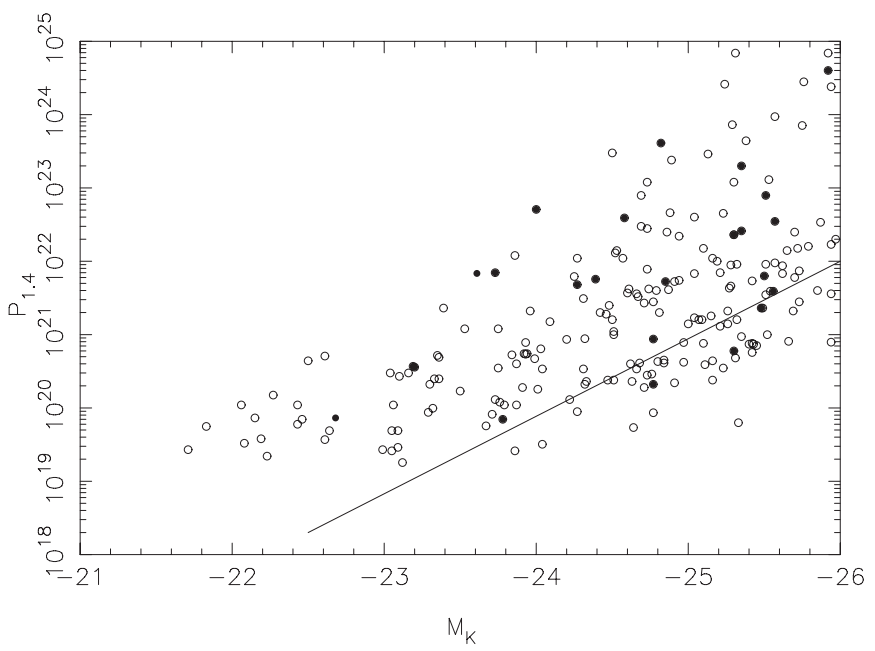

Figure 6. Radio power $\left(\mathrm{W} \mathrm{Hz}^{-1}\right)$ at $1.4 \mathrm{GHz}$ vs. absolute $K$ magnitude. The data are from Brown et al. (2011). Galaxies whose radio powers are upper limits are not distinguished. Emission-line nuclei are denoted by solid symbols. The line is from the original reference and is the median radio power as a function of $K$-band luminosity for all ellipticals (including those not detected by NVSS). As this sample only includes radio sources, it is automatically biased toward higher radio powers and is systematically above the line.

which in turn is responsible for more radio power. Indeed, Ho (2008) shows a strong correlation between radio loudness and $\mathrm{X}$-ray luminosity divided by Eddington ratio. However, if our infrared emission-line galaxies stand out, it may be because the $\mathrm{BH}$ in these cases is currently being fed by the circumnuclear disk.
We also examined the thermal infrared fluxes of the emissionline galaxies. Only UGC 3426 and NGC 3516 had mid-infrared excesses in the AKARI Point Source Catalog (Murakami et al. 2007): The former has a $9 \mu / 2.2 \mu$ flux ratio of 1.7 and the latter 1.0. The infrared flux of UGC 3426 rises a further factor of six at $18 \mu$. In general, the IRAS fluxes of our near-infrared emissionline galaxies do not correlate well with the SFR in Table 2 (Figure 7).

The infrared emission-line spectra in Table 2 are not all clones of one another. We compare the Br $\gamma$ profiles of NGC 2787 and IC 630 in Figure 8. The former has a broad rounded profile, FWHM $750 \mathrm{~km} \mathrm{~s}^{-1}$. The latter has a sharp profile, FWHM $200 \mathrm{~km} \mathrm{~s}^{-1}$. The former is a LINER (Balzano 1983) and the latter is a starburst nucleus in the Veron-Cetty \& Veron (2006) catalog. Both are morphologically classified S0. These differences are a strong motivation to explore the spatial structure of the emitting gas at $10 \mathrm{~m}$ class diffraction limited resolution.

In Figure 9, we show the relation between BH mass and SFR. The latter is calculated from $\mathrm{Br} \gamma$ (Kennicutt 1998). SFR uncertainties were calculated from the $\mathrm{Br} \gamma$ uncertainties in Table 2. We draw the uncertainty in the $\mathrm{BH}$ mass from Table 4 where both the $\left(K, M_{\mathrm{BH}}\right)$ and $\left(\sigma, M_{\mathrm{BH}}\right)$ relations are employed and the galaxies have secondary distance indicators. It is largely a scatter plot, although the sample is modest, and there is a hint of a correlation in the galaxy scaling sense. If the $\mathrm{BH}$ formation process were steady in solar masses/year, one might expect a correlation. If the process is sporadic, dependent, for example, on fueling events or circumnuclear disk instability, a correlation would easily be masked. Monitoring $\mathrm{Br} \gamma$ over time or high-resolution analysis of any disk could shed light on this. 
Table 2

Emission Line Nuclei

\begin{tabular}{|c|c|c|c|c|c|c|c|c|}
\hline NAME & $\begin{array}{l}\text { R.A. } \\
(2000)\end{array}$ & Decl. & Run & $\begin{array}{c}\mathrm{Br} \gamma \text { Flux } \\
\left(\mathrm{erg} \mathrm{cm}^{-2} \mathrm{~s}^{-1}\right)\end{array}$ & $\begin{array}{c}\text { SFR } \\
\left(M_{\odot} \mathrm{yr}^{-1}\right)\end{array}$ & Remarks & $\begin{array}{c}P_{1.4} \\
\left(\mathrm{~W} \mathrm{~Hz}^{-1}\right)\end{array}$ & Redshift \\
\hline NGC 128 & $00: 29: 15$ & $+02: 51: 51$ & 1 & $1.11 \pm 0.04 \mathrm{E}-15$ & 0.38 & $1.74 \mu$ unident. & $6.0 \mathrm{E}+20$ & 0.014 \\
\hline NGC 524 & 01:24:48 & $+09: 32: 19$ & 1 & $1.85 \pm 0.06 \mathrm{E}-15$ & 0.18 & very broad $1.6 \mu$ & $2.1 \mathrm{E}+20$ & 0.008 \\
\hline NGC 547 & 01:26:01 & $-01: 20: 42$ & 1 & $3.97 \pm 0.04 \mathrm{E}-16$ & 0.18 & & $4.0 \mathrm{E}+24$ & 0.018 \\
\hline NGC 665 & 01:44:56 & $+10: 25: 23$ & 1 & $6.92 \pm 0.03 \mathrm{E}-16$ & 0.38 & & $6.3 \mathrm{E}+21$ & 0.018 \\
\hline NGC 708 & $01: 52: 46$ & $+36: 09: 06$ & 1 & $9.1 \pm 0.9 \mathrm{E}-16$ & 0.37 & & $3.5 \mathrm{E}+22$ & 0.016 \\
\hline NGC 1052 & $02: 41: 05$ & $-08: 15: 21$ & 1 & & & $1.26,1.64 \mu[\mathrm{Fe} \mathrm{II}]$ & $5.1 \mathrm{E}+22$ & 0.005 \\
\hline NGC 1521 & 04:08:19 & $-21: 03: 07$ & 3 & $4.62 \pm 0.05 \mathrm{E}-15$ & 1.5 & & $2.3 E+21$ & 0.014 \\
\hline NGC 1550 & 04:19:38 & $+02: 24: 35$ & 1 & $1.27 \pm 0.03 \mathrm{E}-15$ & 0.31 & & $5.3 \mathrm{E}+21$ & 0.012 \\
\hline NGC 2110 & $05: 52: 11$ & $-07: 27: 22$ & 1 & & & & $3.9 \mathrm{E}+22$ & 0.008 \\
\hline NGC 2128 & 06:04:34 & $+57: 37: 40$ & 1 & $1.7 \pm 0.1 \mathrm{E}-15$ & 0.31 & & $5.7 \mathrm{E}+21$ & 0.010 \\
\hline NGC 2273 & 06:50:08 & $+60: 50: 45$ & 4 & & & [Fe II] lines & $6.8 \mathrm{E}+21$ & 0.006 \\
\hline UGC3426 & $06: 15: 36$ & $+71: 02: 15$ & 1 & $1.2 \pm 0.02 \mathrm{E}-13$ & 37.2 & & $4.1 \mathrm{E}+23$ & 0.0135 \\
\hline NGC 2768 & $09: 11: 38$ & $+60: 02: 14$ & 1 & & & & $8.7 \mathrm{E}+20$ & 0.004 \\
\hline NGC 2787 & 09:19:18 & $+69: 12: 12$ & 1 & $1.63 \pm 0.05 \mathrm{E}-14$ & 0.31 & & $7.3 \mathrm{E}+19$ & 0.002 \\
\hline NGC 2859 & 09:24:19 & $+34: 30: 49$ & 1 & $7.7 \pm 0.4 \mathrm{E}-15$ & 0.49 & & $7.0 \mathrm{E}+19$ & 0.006 \\
\hline NGC 3065 & 10:01:55 & $+72: 10: 13$ & 1 & $1.8 \pm 0.1 \mathrm{E}-15$ & 0.18 & & $3.6 \mathrm{E}+20$ & 0.007 \\
\hline IC630 & $10: 38: 33$ & $-07: 10: 14$ & 3 & $1.99 \pm 0.01 \mathrm{E}-14$ & 2.1 & & $7.0 \mathrm{E}+21$ & 0.007 \\
\hline NGC 3516 & 11:06:47 & $+72: 34: 07$ & 3 & & & Broad $\mathrm{P} \beta$ & $4.8 \mathrm{E}+21$ & 0.009 \\
\hline NGC 4111 & 12:07:03.1 & $+43: 03: 57$ & 5 & $1.5 \pm 0.1 \mathrm{E}-15$ & 0.04 & + & $2.5 \mathrm{E}+20$ & 0.003 \\
\hline NGC 5273 & $13: 42: 08.3$ & $+35: 39: 15$ & 5 & & & Broad $\mathrm{P} \gamma$ & $1.1 \mathrm{E}+20$ & 0.004 \\
\hline NGC 5322 & $13: 49: 15.3$ & $+60: 11: 26$ & 5 & $1.9 \pm 0.8 \mathrm{E}-15$ & 0.18 & & $9.1 \mathrm{E}+21$ & 0.006 \\
\hline NGC 7052 & $21: 18: 33$ & $+26: 26: 49$ & 1 & $2.49 \pm 0.07 \mathrm{E}-15$ & 1.03 & & $7.9 \mathrm{E}+22$ & 0.016 \\
\hline NGC 7426 & $22: 56: 03$ & $+36: 21: 41$ & 1 & $3.75 \pm 0.09 \mathrm{E}-15$ & 2.01 & & $3.9 \mathrm{E}+21$ & 0.018 \\
\hline NGC 7550 & $23: 15: 16$ & $+18: 57: 42$ & 1 & $1.77 \pm 0.04 \mathrm{E}-15$ & 0.86 & & $2.6 \mathrm{E}+22$ & 0.017 \\
\hline NGC 7618 & $23: 19: 47$ & $+42: 51: 10$ & 1 & $1.94 \pm 0.04 \mathrm{E}-15$ & 0.99 & & $2.3 \mathrm{E}+22$ & 0.017 \\
\hline NGC 7626 & $23: 20: 43$ & $+08: 13: 01$ & 1 & $1.86 \pm 0.06 \mathrm{E}-15$ & 0.43 & & $2.0 \mathrm{E}+23$ & 0.011 \\
\hline NGC 7743 & $23: 44: 21$ & $+09: 56: 03$ & 1 & $1.90 \pm 0.07 \mathrm{E}-15$ & 0.09 & & $3.7 \mathrm{E}+20$ & 0.006 \\
\hline
\end{tabular}

Notes. The remark on broad Paschen lines applies to all four of NGC 3516, 3557, 4111, and 5273. The redshifts in Column 9 are the literature, not observed, values.

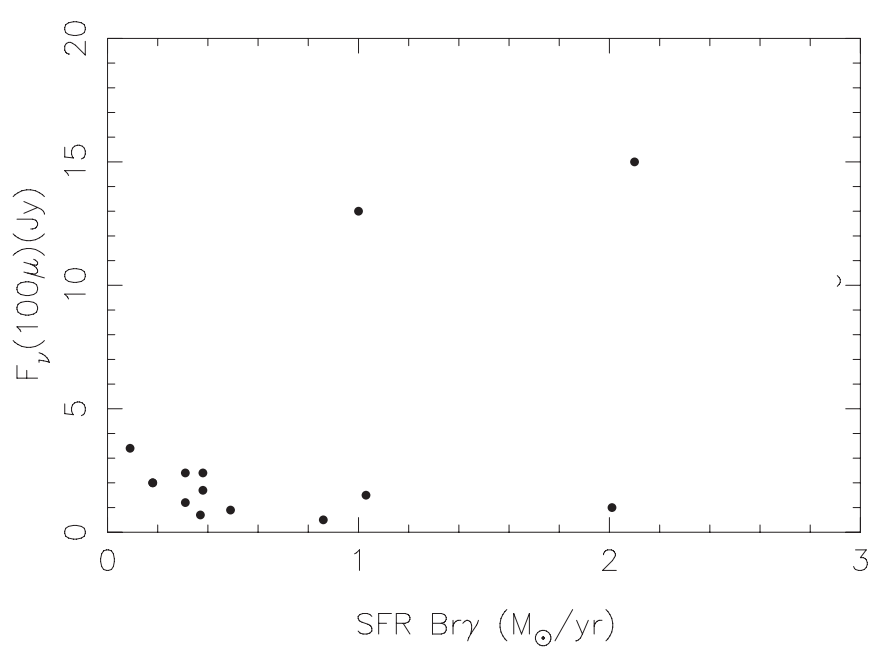

Figure 7. Comparison of the $\mathrm{Br} \gamma$ star formation rates with IRAS far infrared fluxes. UGC 3426 is the upper limit at $10 \mathrm{Jy}$.
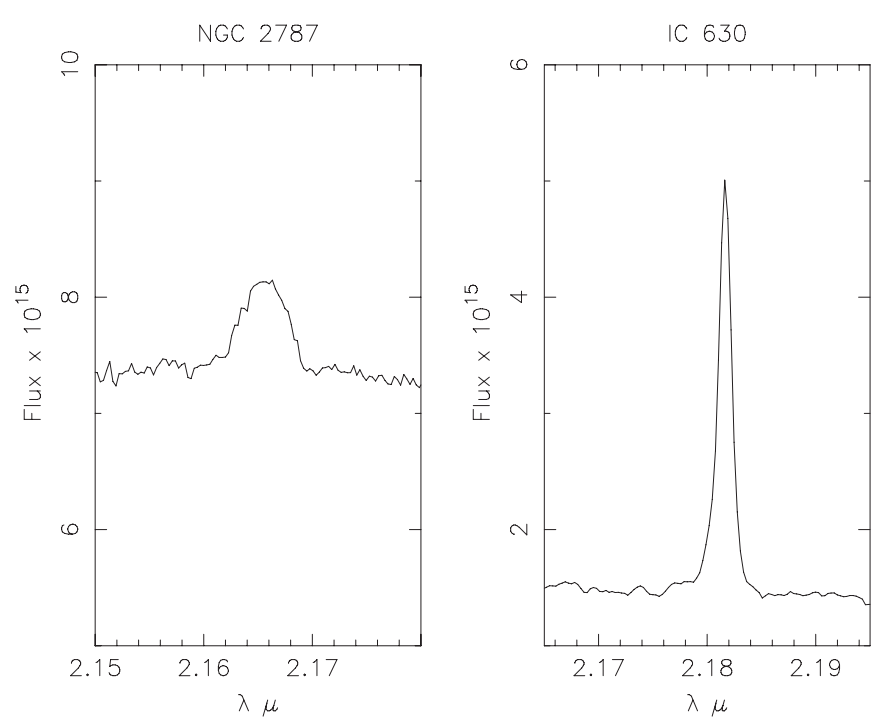

Figure 8. Comparison of the $\mathrm{Br} \gamma$ profiles of two galaxies. The spectra have not been corrected to the rest frame. 
Table 3

Absorption Line Nuclei

\begin{tabular}{|c|c|c|c|c|c|}
\hline NAME & Run & $\mathrm{S} / \mathrm{N}$ & NAME & Run & $\mathrm{S} / \mathrm{N}$ \\
\hline NGC 16 & 1 & 93 & NGC 3091 & 4 & 3 \\
\hline NGC 50 & 2 & 8 & NGC 3100 & 3 & \\
\hline NGC 57 & 4 & 14 & NGC 3158 & 2 & 23 \\
\hline NGC 315 & 1 & 50 & NGC 3226 & 2 & 22 \\
\hline NGC 383 & 1 & & NGC 3245 & 2 & 24 \\
\hline NGC 410 & 1 & 22 & NGC 3348 & 4 & 8 \\
\hline NGC 439 & 3 & & NGC 7454 & 2 & 10 \\
\hline NGC 474 & 1 & 49 & NGC 3414 & 2 & 30 \\
\hline NGC 499 & 4 & 12 & NGC 3528 & 4 & 20 \\
\hline NGC 533 & 2 & 2 & NGC 3607 & 4 & 38 \\
\hline NGC 596 & 4 & 20 & NGC 3619 & 4 & 26 \\
\hline NGC 680 & 4 & 13 & NGC 3626 & 4 & 14 \\
\hline NGC 741 & 2 & 7 & NGC 3665 & 4 & 34 \\
\hline NGC 883 & 2 & 7 & NGC 3801 & 4 & 7 \\
\hline NGC 936 & 2 & 10 & NGC 3872 & 4 & 17 \\
\hline NGC 1016 & 2 & 2 & NGC 3894 & 4 & 20 \\
\hline NGC 1060 & 2 & 15 & NGC 3957 & 4 & 11 \\
\hline NGC 1128 & 1 & 70 & NGC 3962 & 4 & 31 \\
\hline NGC 1167 & 1 & 52 & NGC 3986 & 4 & 14 \\
\hline NGC 1200 & 2 & & NGC 4008 & 4 & 5 \\
\hline NGC 1209 & 4 & 10 & NGC 4036 & 4 & 31 \\
\hline NGC 1326 & 3 & 74 & NGC 4125 & 4 & 31 \\
\hline NGC 1399 & 3 & 95 & NGC 4138 & 4 & 28 \\
\hline NGC 1400 & 2 & & NGC 4220 & 4 & 17 \\
\hline NGC 1407 & 2 & 8 & NGC 4589 & 4 & 22 \\
\hline NGC 1453 & 1 & 89 & NGC 4636 & 4 & 25 \\
\hline NGC 1531 & 3 & 15 & NGC 4778 & 4 & 8 \\
\hline IC359 & 2 & & NGC 4782 & 4 & 17 \\
\hline UGC3024 & 2 & 6 & NGC 4802 & 4 & 15 \\
\hline NGC 1587 & 1 & 64 & NGC 4825 & 4 & 19 \\
\hline NGC 1600 & 2 & 8 & NGC 4874 & 4 & 8 \\
\hline NGC 1573 & 2 & 10 & NGC 4984 & 4 & 28 \\
\hline NGC 1653 & 1 & 70 & NGC 5044 & 4 & 6 \\
\hline NGC 1684 & 1 & 68 & NGC 5077 & 4 & 16 \\
\hline NGC 1726 & 2 & 10 & NGC 5198 & 4 & 20 \\
\hline NGC 2089 & 2 & 8 & NGC 5444 & 4 & 23 \\
\hline NGC 2208 & 2 & 4 & NGC 5490 & 4 & 21 \\
\hline NGC 2256 & 4 & 4 & NGC 5532 & 4 & 9 \\
\hline NGC 2258 & 2 & 28 & NGC 5631 & 4 & 21 \\
\hline NGC 2320 & 4 & 15 & NGC 5838 & 4 & 16 \\
\hline NGC 2314 & 4 & 9 & IC 459 & 3 & 26 \\
\hline NGC 2340 & 2 & 8 & NGC 5846 & 1 & 32 \\
\hline NGC 2418 & 4 & 20 & NGC 6251 & 1 & 53 \\
\hline NGC 2493 & 3 & 85 & NGC 6482 & 1 & 49 \\
\hline NGC 2513 & 2 & 30 & NGC 6703 & 1 & 67 \\
\hline NGC 2612 & 4 & 24 & NGC 6869 & 2 & 8 \\
\hline UGC3789 & 4 & 15 & NGC 6903 & 2 & 8 \\
\hline NGC 2629 & 3 & 71 & NGC 7242 & 2 & 7 \\
\hline NGC 2685 & 1 & 47 & NGC 7265 & 2 & 13 \\
\hline NGC 2693 & 3 & 82 & NGC 7391 & 2 & 11 \\
\hline NGC 2749 & 2 & 23 & NGC 7436 & 2 & 22 \\
\hline NGC 2872 & 3 & 78 & NGC 7562 & 2 & 7 \\
\hline NGC 2911 & 1 & 19 & NGC 7600 & 2 & 9 \\
\hline NGC 2974 & 4 & 55 & NGC 7711 & 1 & 83 \\
\hline NGC 3078 & 3 & 103 & NGC 7785 & 1 & 69 \\
\hline NGC 4551 & 5 & 50 & NGC 5796 & 4 & 23 \\
\hline NGC 5813 & 5 & 54 & NGC 4143 & 5 & 87 \\
\hline NGC 4203 & 5 & 97 & NGC 4150 & 5 & 64 \\
\hline NGC 4278 & 5 & 83 & NGC 4710 & 5 & 29 \\
\hline NGC 4866 & 5 & 47 & NGC 5353 & 5 & 93 \\
\hline
\end{tabular}


Table 4

BH Masses For Brown et al. (2011) Galaxies

\begin{tabular}{|c|c|c|c|c|c|c|c|c|c|}
\hline Name & $\begin{array}{l}\text { R.A. }^{\mathrm{a}} \\
(\mathrm{J} 2000)\end{array}$ & Decl. & $\begin{array}{c}m-M^{\mathrm{b}} \\
(\mathrm{mag})\end{array}$ & Method $^{\mathrm{c}}$ & $\begin{array}{c}K^{\mathrm{d}} \\
(2 \mathrm{MASS})\end{array}$ & $\begin{array}{c}\sigma^{\mathrm{e}} \\
\left(\mathrm{km} \mathrm{s}^{-1}\right)\end{array}$ & $\begin{array}{l}M(\mathrm{BH})_{K}{ }^{\mathrm{f}} \\
\left(10^{8} M_{\odot}\right)\end{array}$ & $\begin{array}{l}M(\mathrm{BH})_{\sigma}{ }^{\mathrm{g}} \\
\left(10^{8} M_{\odot}\right)\end{array}$ & Notes $^{h}$ \\
\hline NGC 0016 & 00:09:04 & $+27: 43: 45$ & 33.2 & $\mathrm{CV}$ & 8.78 & 180 & 3.18 & 0.99 & \\
\hline NGC 0050 & $00: 14: 44$ & $-07: 20: 42$ & 34.4 & $\mathrm{CV}$ & 8.67 & 264 & 11.77 & 6.40 & \\
\hline NGC 0057 & $00: 15: 30$ & $+17: 19: 42$ & 34.4 & $\mathrm{CV}$ & 8.68 & 326 & 11.41 & 17.84 & \\
\hline NGC 0128 & $00: 29: 15$ & $+02: 51: 50$ & 33.9 & $\mathrm{CV}$ & 8.52 & 215 & 7.90 & 2.36 & \\
\hline NGC 0315 & $00: 57: 48$ & $+30: 21: 29$ & 34.2 & $\mathrm{CV}$ & 7.95 & 279 & 18.89 & 8.37 & \\
\hline NGC 0383 & $01: 07: 24$ & $+32: 24: 44$ & 34.2 & $\mathrm{CV}$ & 8.48 & 279 & 12.10 & 8.37 & \\
\hline NGC 0410 & $01: 10: 58$ & $+33: 09: 08$ & 34.3 & $\mathrm{CV}$ & 8.38 & 300 & 14.41 & 11.91 & \\
\hline NGC 0439 & $01: 13: 47$ & $-31: 44: 49$ & 34.5 & $\mathrm{CV}$ & 8.70 & 221 & 12.65 & 2.70 & \\
\hline NGC 0474 & 01:20:06 & $+03: 24: 55$ & 32.5 & $\mathrm{CV}$ & 8.55 & 163 & 2.18 & 0.61 & \\
\hline NGC 0499 & $01: 23: 11$ & $+33: 27: 36$ & 33.9 & $\mathrm{CV}$ & 8.73 & 266 & 6.94 & 6.64 & \\
\hline NGC 0524 & $01: 24: 47$ & $+09: 32: 19$ & 32.6 & $\mathrm{CV}$ & 7.16 & 253 & 8.77 & 5.20 & \\
\hline NGC 0533 & $01: 25: 31$ & $+01: 45: 32$ & 34.4 & $\mathrm{CV}$ & 8.44 & 279 & 14.92 & 8.37 & \\
\hline NGC 0547 & $01: 26: 00$ & $-01: 20: 42$ & 34.4 & $\mathrm{CV}$ & 8.49 & 260 & 13.85 & 5.94 & \\
\hline NGC 0596 & $01: 32: 51$ & $-07: 01: 53$ & 32.1 & $\mathrm{CV}$ & 7.97 & 152 & 2.40 & 0.44 & \\
\hline NGC 0665 & $01: 44: 56$ & $+10: 25: 23$ & 34.4 & $\mathrm{CV}$ & 8.88 & 190 & 9.37 & 1.29 & \\
\hline NGC 0680 & $01: 49: 47$ & $+21: 58: 14$ & 33.0 & $\mathrm{CV}$ & 8.73 & 212 & 2.70 & 2.20 & \\
\hline NGC 0708 & $01: 52: 46$ & $+36: 09: 07$ & 34.1 & $\mathrm{CV}$ & 8.57 & 230 & 9.94 & 3.27 & \\
\hline NGC 0741 & $01: 56: 20$ & $+05: 37: 43$ & 34.4 & $\mathrm{CV}$ & 8.29 & 291 & 17.40 & 10.27 & \\
\hline NGC 0883 & 02:19:05 & $-06: 47: 27$ & 34.3 & $\mathrm{CV}$ & 8.89 & 287 & 8.84 & 9.60 & \\
\hline NGC 0936 & $02: 27: 37$ & $-01: 09: 21$ & 31.4 & $\mathrm{CV}$ & 6.91 & 189 & 3.34 & 1.26 & \\
\hline NGC 1016 & $02: 38: 19$ & $+02: 07: 09$ & 34.1 & $D_{n}-\sigma$ & 8.58 & 302 & 10.02 & 12.30 & \\
\hline NGC 1052 & 02:41:04 & $-08: 15: 20$ & 31.4 & SBF & 7.45 & 207 & 2.16 & 1.96 & \\
\hline NGC 1060 & $02: 43: 15$ & $+32: 25: 29$ & 34.3 & $D_{n}-\sigma$ & 8.20 & 303 & 16.18 & 12.50 & \\
\hline NGC 1128 & $02: 57: 42$ & $+06: 01: 29$ & 34.9 & $\mathrm{CV}$ & 8.98 & $\ldots$ & 14.72 & $\ldots$ & \\
\hline NGC 1167 & $03: 01: 42$ & $+35: 12: 20$ & 34.2 & $\mathrm{CV}$ & 8.64 & 171 & 9.68 & 0.78 & \\
\hline NGC 1200 & $03: 03: 54$ & $-11: 59: 29$ & 33.7 & $\mathrm{CV}$ & 8.58 & 204 & 6.56 & 1.83 & \\
\hline NGC 1209 & 03:06:02 & $-15: 36: 40$ & 32.8 & SBF & 8.32 & 230 & 3.37 & 3.27 & \\
\hline NGC 1316 & $03: 22: 41$ & $-37: 12: 29$ & 31.7 & SBF & 5.59 & 228 & 16.23 & 3.14 & \\
\hline NGC 1326 & $03: 23: 56$ & $-36: 27: 52$ & 31.4 & $\mathrm{TF}$ & 7.45 & 118 & 2.04 & 0.13 & \\
\hline NGC 1399 & $03: 38: 29$ & $-35: 27: 02$ & 31.6 & SBF & 6.31 & 346 & 7.64 & $23.85 .1 \pm 0.7$ & (3) \\
\hline NGC 1400 & $03: 39: 30$ & $-18: 41: 17$ & 31.1 & SBF & 7.81 & 252 & 1.06 & 5.10 & \\
\hline NGC 1407 & $03: 40: 11$ & $-18: 34: 48$ & 31.1 & $\mathrm{SBF}$ & 6.70 & 271 & 3.12 & 7.27 & \\
\hline NGC 1453 & $03: 46: 27$ & $-03: 58: 08$ & 33.7 & $D_{n}-\sigma$ & 8.12 & 328 & 10.38 & 18.38 & \\
\hline NGC 1521 & 04:08:18 & $-21: 03: 06$ & 34.1 & SBF & 8.68 & 242 & 8.98 & 4.19 & \\
\hline NGC 1531 & $04: 11: 59$ & $-32: 51: 03$ & 30.6 & $\mathrm{TF}$ & 8.42 & 112 & 0.36 & 0.10 & \\
\hline IC0359 & $04: 12: 28$ & $+27: 42: 06$ & 33.8 & $\mathrm{CV}$ & 8.47 & $\ldots$ & 7.54 & $\ldots$ & \\
\hline NGC 1550 & $04: 19: 37$ & $+02: 24: 35$ & 33.6 & $\mathrm{CV}$ & 8.77 & 308 & 4.68 & 13.54 & \\
\hline UGC3024 & $04: 22: 26$ & $+27: 17: 52$ & 34.3 & $\mathrm{CV}$ & 9.25 & $\ldots$ & 6.10 & $\ldots$ & \\
\hline NGC 1587 & $04: 30: 39$ & $+00: 39: 42$ & 32.8 & $D_{n}-\sigma$ & 8.51 & 227 & 2.86 & 3.07 & \\
\hline NGC 1600 & $04: 31: 39$ & $-05: 05: 09$ & 33.5 & $D_{n}-\sigma$ & 8.04 & 334 & 9.41 & 20.07 & \\
\hline NGC 1573 & $04: 35: 03$ & $+73: 15: 44$ & 33.9 & $D_{n}-\sigma$ & 8.55 & 303 & 7.80 & 12.50 & \\
\hline NGC 1653 & $04: 45: 47$ & $-02: 23: 33$ & 33.8 & $\mathrm{SBF}$ & 8.97 & 246 & 4.95 & 4.54 & \\
\hline NGC 1684 & $04: 52: 31$ & $-03: 06: 21$ & 33.9 & $\mathrm{CV}$ & 8.69 & 306 & 7.25 & 13.11 & \\
\hline NGC 1726 & $04: 59: 41$ & $-07: 45: 19$ & 33.0 & $D_{n}-\sigma$ & 8.61 & 246 & 3.20 & 4.54 & \\
\hline NGC 2089 & $05: 47: 51$ & $-17: 36: 09$ & 33.0 & $\mathrm{CV}$ & 8.81 & 206 & 2.57 & 1.92 & \\
\hline NGC 2110 & $05: 52: 11$ & $-07: 27: 22$ & 32.6 & $\mathrm{CV}$ & 8.14 & 255 & 3.46 & 5.41 & \\
\hline NGC 2128 & $06: 04: 34$ & $+57: 37: 40$ & 33.1 & $\mathrm{CV}$ & 8.83 & 261 & 2.87 & 6.05 & \\
\hline UGC3426 & $06: 15: 36$ & $+71: 02: 15$ & 33.8 & $\mathrm{CV}$ & 8.97 & 283 & 4.65 & 8.97 & \\
\hline NGC 2208 & $06: 22: 34$ & $+51: 54: 34$ & 34.5 & $\mathrm{CV}$ & 9.04 & 225 & 9.34 & 2.94 & \\
\hline NGC 2256 & $06: 47: 13$ & $+74: 14: 11$ & 34.3 & $\mathrm{CV}$ & 8.67 & 221 & 10.73 & 2.70 & \\
\hline NGC 2258 & $06: 47: 46$ & $+74: 28: 54$ & 33.6 & SBF & 8.23 & 287 & 7.93 & 9.60 & \\
\hline NGC 2273 & 06:50:08 & $+60: 50: 44$ & 32.3 & $\mathrm{TF}$ & 8.48 & 123 & 1.77 & 0.16 & \\
\hline NGC 2273 & 06:50:08 & $+60: 50: 44$ & 32.3 & $\mathrm{TF}$ & 8.48 & 123 & 1.77 & 0.16 & \\
\hline NGC 2320 & $07: 05: 41$ & $+50: 34: 51$ & 34.5 & SNIa & 8.85 & 315 & 10.69 & 15.10 & \\
\hline NGC 2314 & $07: 10: 32$ & $+75: 19: 36$ & 34.0 & $D_{n}-\sigma$ & 8.88 & 286 & 6.70 & 9.44 & \\
\hline IC0459 & $07: 10: 38$ & $+50: 10: 37$ & 34.5 & $\mathrm{CV}$ & 11.29 & $\ldots$ & 0.99 & $\ldots$ & \\
\hline NGC 2340 & $07: 11: 10$ & $+50: 10: 28$ & 34.7 & $D_{n}-\sigma$ & 8.88 & 246 & 13.18 & 4.54 & \\
\hline UGC3789 & $07: 19: 30$ & $+59: 21: 18$ & 33.3 & $\mathrm{CV}$ & 9.51 & $\ldots$ & 1.75 & $\ldots$ & \\
\hline NGC 2418 & $07: 36: 37$ & $+17: 53: 02$ & 34.2 & $\mathrm{CV}$ & 8.95 & 247 & 7.50 & 4.63 & \\
\hline NGC 2493 & 08:00:23 & $+39: 49: 49$ & 33.7 & $\mathrm{CV}$ & 8.83 & 249 & 4.94 & 4.82 & \\
\hline NGC 2513 & $08: 02: 24$ & $+09: 24: 47$ & 33.9 & $D_{n}-\sigma$ & 8.74 & 274 & 6.51 & 7.67 & \\
\hline NGC 2612 & $08: 33: 50$ & $-13: 10: 28$ & 31.8 & $\mathrm{CV}$ & 8.78 & $\ldots$ & 0.83 & $\ldots$ & \\
\hline NGC 2629 & $08: 47: 15$ & $+72: 59: 08$ & 33.6 & $\mathrm{CV}$ & 8.85 & 298 & 4.37 & 11.53 & \\
\hline NGC 2685 & $08: 55: 34$ & $+58: 44: 03$ & 30.9 & $\mathrm{TF}$ & 8.35 & 94 & 0.54 & 0.04 & \\
\hline NGC 2693 & $08: 56: 59$ & $+51: 20: 50$ & 34.0 & $D_{n}-\sigma$ & 8.60 & 349 & 8.27 & 24.84 & \\
\hline
\end{tabular}


Table 4

(Continued)

\begin{tabular}{|c|c|c|c|c|c|c|c|c|c|}
\hline Name & $\begin{array}{l}\text { R.A. }^{\mathrm{a}} \\
\text { (J2000) }\end{array}$ & Decl. & $\begin{array}{c}m-M^{\mathrm{b}} \\
(\mathrm{mag})\end{array}$ & Method $^{\mathrm{c}}$ & $\begin{array}{c}K^{\mathrm{d}} \\
(2 \mathrm{MASS})\end{array}$ & $\begin{array}{c}\sigma^{\mathrm{e}} \\
\left(\mathrm{km} \mathrm{s}^{-1}\right)\end{array}$ & $\begin{array}{l}M(\mathrm{BH})_{K}{ }^{\mathrm{f}} \\
\left(10^{8} M_{\odot}\right)\end{array}$ & $\begin{array}{l}M(\mathrm{BH})_{\sigma} \mathrm{g} \\
\left(10^{8} M_{\odot}\right)\end{array}$ & Notes ${ }^{h}$ \\
\hline NGC 2749 & 09:05:21 & $+18: 18: 47$ & 33.9 & $D_{n}-\sigma$ & 8.93 & 260 & 5.50 & 5.94 & \\
\hline NGC 2768 & 09:11:37 & $+60: 02: 14$ & 31.8 & $\mathrm{SBF}$ & 7.00 & 181 & 4.53 & 1.02 & \\
\hline NGC 2787 & 09:19:18 & $+69: 12: 11$ & 29.4 & SBF & 7.26 & 194 & 0.35 & $1.431 .04_{-0.64}^{+0.36}$ & (1) \\
\hline NGC 2859 & $09: 24: 18$ & $+34: 30: 48$ & 32.0 & $\mathrm{TF}$ & 8.04 & 181 & 2.17 & 1.02 & \\
\hline NGC 2872 & $09: 25: 42$ & $+11: 25: 55$ & 33.9 & $D_{n}-\sigma$ & 8.72 & 284 & 6.75 & 9.12 & \\
\hline NGC 2911 & 09:33:46 & $+10: 09: 07$ & 33.3 & $\mathrm{CV}$ & 8.71 & 217 & 3.72 & 2.47 & \\
\hline NGC 2974 & $09: 42: 33$ & $-03: 41: 57$ & 31.7 & SBF & 6.25 & 238 & 8.51 & 3.87 & \\
\hline NGC 3078 & 09:58:24 & $-26: 55: 33$ & 32.7 & SBF & 7.88 & 251 & 4.97 & 5.01 & \\
\hline NGC 3091 & 10:00:14 & $-19: 38: 10$ & 33.6 & $D_{n}-\sigma$ & 8.09 & 321 & 9.20 & 16.55 & \\
\hline NGC 3100 & 10:00:40 & $-31: 39: 51$ & 32.8 & $\mathrm{CV}$ & 8.08 & 200 & 4.27 & 1.66 & \\
\hline NGC 3065 & 10:01:55 & $+72: 10: 13$ & 32.5 & $\mathrm{TF}$ & 8.99 & 160 & 1.34 & 0.56 & \\
\hline NGC 3158 & $10: 13: 50$ & $+38: 45: 53$ & 34.5 & $D_{n}-\sigma$ & 8.80 & 343 & 11.47 & 22.84 & \\
\hline NGC 3226 & $10: 23: 26$ & $+19: 53: 53$ & 31.9 & $\mathrm{SBF}$ & 8.57 & 193 & 1.10 & 1.40 & \\
\hline NGC 3245 & $10: 27: 18$ & $+28: 30: 26$ & 31.6 & SBF & 7.86 & 210 & 1.70 & $2.102 .04 \pm 0.49$ & (4) \\
\hline IC0630 & $10: 38: 33$ & $-07: 10: 14$ & 32.4 & $\mathrm{CV}$ & 8.65 & $\ldots$ & 1.73 & $\ldots$ & \\
\hline NGC 3348 & 10:47:09 & $+72: 50: 22$ & 32.7 & $D_{n}-\sigma$ & 7.96 & 238 & 4.45 & 3.87 & \\
\hline NGC 3414 & $10: 51: 16$ & $+27: 58: 28$ & 32.0 & $\mathrm{SBF}$ & 7.98 & 237 & 2.25 & 3.79 & \\
\hline NGC 3516 & 11:06:47 & $+72: 34: 06$ & 33.0 & $\mathrm{TF}$ & 8.51 & 157 & 3.34 & 0.51 & \\
\hline NGC 3497 & 11:07:18 & $-19: 28: 17$ & 33.5 & $\mathrm{CV}$ & 8.67 & 224 & 5.02 & $2.88==$ NGC 3528 & \\
\hline NGC 3607 & $11: 16: 54$ & $+18: 03: 09$ & 31.8 & SBF & 6.99 & 224 & 4.74 & $2.881 .33 \pm 0.44$ & (5) \\
\hline NGC 3619 & $11: 19: 21$ & $+57: 45: 28$ & 32.2 & $\mathrm{TF}$ & 8.57 & 162 & 1.57 & 0.60 & \\
\hline NGC 3626 & 11:20:03 & $+18: 21: 26$ & 31.5 & SBF & 8.16 & 142 & 1.17 & 0.31 & \\
\hline NGC 3665 & $11: 24: 43$ & $+38: 45: 46$ & 32.6 & $\mathrm{TF}$ & 7.68 & 184 & 5.11 & 1.11 & \\
\hline NGC 3801 & $11: 40: 16$ & $+17: 43: 41$ & 33.4 & $\mathrm{CV}$ & 8.88 & 198 & 3.67 & 1.58 & \\
\hline NGC 3872 & $11: 45: 49$ & $+13: 46: 00$ & 33.3 & $D_{n}-\sigma$ & 8.51 & 260 & 4.75 & 5.94 & \\
\hline NGC 3894 & $11: 48: 50$ & $+59: 24: 56$ & 33.2 & $\mathrm{CV}$ & 8.56 & 265 & 4.28 & 6.52 & \\
\hline NGC 3957 & 11:54:01 & $-19: 34: 06$ & 32.2 & $\mathrm{TF}$ & 8.69 & & 1.36 & $\ldots$ & \\
\hline NGC 3962 & $11: 54: 40$ & $-13: 58: 29$ & 32.7 & SBF & 7.67 & 233 & 6.17 & 3.49 & \\
\hline NGC 3986 & $11: 56: 44$ & $+32: 01: 18$ & 33.3 & $\mathrm{CV}$ & 8.98 & 196 & 2.89 & 1.50 & \\
\hline NGC 4008 & $11: 58: 17$ & $+28: 11: 32$ & 33.4 & $D_{n}-\sigma$ & 8.83 & 226 & 3.65 & 3.01 & \\
\hline NGC 4036 & $12: 01: 26$ & $+61: 53: 44$ & 31.9 & $\mathrm{TF}$ & 7.56 & 181 & 3.18 & 1.02 & \\
\hline NGC 4125 & 12:08:06 & $+65: 10: 26$ & 31.9 & SBF & 6.86 & 227 & 5.95 & 3.07 & \\
\hline NGC 4138 & $12: 09: 29$ & $+43: 41: 06$ & 30.7 & SBF & 8.20 & 140 & 0.51 & 0.29 & \\
\hline NGC 4220 & $12: 16: 11$ & $+47: 52: 59$ & 31.4 & $\mathrm{TF}$ & 8.13 & 125 & 1.05 & 0.17 & \\
\hline NGC 4589 & $12: 37: 25$ & $+74: 11: 30$ & 31.7 & SBF & 7.76 & 224 & 2.09 & 2.88 & \\
\hline NGC 4636 & $12: 42: 49$ & $+02: 41: 15$ & 30.8 & SBF & 6.42 & 203 & 3.24 & 1.78 & \\
\hline NGC 4778 & $12: 53: 06$ & $-09: 12: 14$ & 33.9 & $\mathrm{CV}$ & 8.63 & 232 & 7.35 & $3.42==$ NGC 4759 & \\
\hline NGC 4782 & $12: 54: 35$ & $-12: 34: 06$ & 33.9 & $D_{n}-\sigma$ & 7.75 & 326 & 16.64 & 17.84 & \\
\hline NGC 4802 & $12: 55: 49$ & $-12: 03: 18$ & 30.3 & $\mathrm{SBF}$ & 8.50 & $\ldots$ & 0.26 & $\ldots$ & \\
\hline NGC 4825 & $12: 57: 12$ & $-13: 39: 52$ & 33.9 & $\mathrm{CV}$ & 8.51 & 308 & 8.51 & 13.54 & \\
\hline NGC 4874 & $12: 59: 35$ & $+27: 57: 34$ & 35.0 & GCLF & 8.86 & 279 & 17.49 & 8.37 & \\
\hline NGC 4984 & 13:08:57 & $-15: 30: 58$ & 31.6 & $\mathrm{TF}$ & 7.74 & $\ldots$ & 1.98 & $\ldots$ & \\
\hline NGC 5044 & $13: 15: 23$ & $-16: 23: 04$ & 32.5 & SBF & 7.71 & 242 & 4.56 & 4.19 & \\
\hline NGC 5077 & $13: 19: 31$ & $-12: 39: 21$ & 32.9 & $D_{n}-\sigma$ & 8.22 & 256 & 4.24 & 5.51 & \\
\hline NGC 5198 & $13: 30: 11$ & $+46: 40: 14$ & 33.4 & $D_{n}-\sigma$ & 8.90 & 196 & 3.49 & 1.50 & \\
\hline NGC 5444 & $14: 03: 24$ & $+35: 07: 55$ & 33.7 & $D_{n}-\sigma$ & 8.84 & 229 & 5.13 & 3.21 & \\
\hline NGC 5490 & 14:09:57 & $+17: 32: 43$ & 34.5 & SNIa & 8.92 & 288 & 10.59 & 9.77 & \\
\hline NGC 5532 & $14: 16: 52$ & $+10: 48: 32$ & 35.1 & $\mathrm{CV}$ & 8.76 & 294 & 20.22 & 10.80 & \\
\hline NGC 5631 & $14: 26: 33$ & $+56: 34: 57$ & 32.2 & SBF & 8.47 & 171 & 1.72 & 0.78 & \\
\hline NGC 5796 & $14: 59: 23$ & $-16: 37: 25$ & 32.9 & $D_{n}-\sigma$ & 8.15 & 273 & 4.36 & 7.53 & \\
\hline NGC 5838 & $15: 05: 26$ & $+02: 05: 58$ & 32.3 & $\mathrm{TF}$ & 7.58 & 266 & 4.26 & 6.64 & \\
\hline NGC 5846 & $15: 06: 29$ & $+01: 36: 20$ & 32.0 & SBF & 6.93 & 239 & 6.01 & 3.95 & \\
\hline NGC 6251 & $16: 32: 31$ & $+82: 32: 16$ & 35.1 & $\mathrm{CV}$ & 9.03 & 325 & 15.63 & $17.576 .0 \pm 0.2$ & (6) \\
\hline NGC 6482 & $17: 51: 48$ & $+23: 04: 18$ & 33.7 & $\mathrm{CV}$ & 8.37 & 308 & 7.82 & 13.54 & \\
\hline NGC 6703 & $18: 47: 18$ & $+45: 33: 01$ & 32.1 & SBF & 8.25 & 178 & 1.95 & 0.94 & \\
\hline NGC 6869 & 20:00:42 & $+66: 13: 39$ & 32.5 & SBF & 8.71 & 166 & 1.83 & 0.67 & \\
\hline NGC 7052 & $21: 18: 33$ & $+26: 26: 48$ & 34.1 & $\mathrm{CV}$ & 8.57 & 284 & 9.20 & $9.123 .7_{-1.5}^{+2.6}$ & (2) \\
\hline NGC 7242 & $22: 15: 39$ & $+37: 17: 55$ & 34.5 & $\mathrm{CV}$ & 8.33 & $\ldots$ & 17.95 & $\ldots^{-1.5}$ & \\
\hline NGC 7265 & $22: 22: 27$ & $+36: 12: 35$ & 34.2 & $\mathrm{CV}$ & 8.69 & 258 & 9.79 & 5.72 & \\
\hline NGC 7391 & $22: 50: 36$ & $-01: 32: 41$ & 34.0 & $D_{n}-\sigma$ & 8.63 & 244 & 8.08 & 4.36 & \\
\hline NGC 7426 & $22: 56: 02$ & $+36: 21: 40$ & 34.3 & $\mathrm{CV}$ & 8.81 & $\ldots$ & 9.55 & $\ldots$ & \\
\hline NGC 7436 & $22: 57: 57$ & $+26: 09: 00$ & 35.0 & $\mathrm{CV}$ & 9.01 & 352 & 15.77 & 25.90 & \\
\hline NGC 7454 & 23:01:06 & $+16: 23: 18$ & 31.9 & SBF & 8.86 & 114 & 0.86 & 0.11 & \\
\hline NGC 7454 & 23:01:06 & $+16: 23: 18$ & 31.9 & SBF & 8.86 & 114 & 0.86 & 0.11 & \\
\hline NGC 7550 & $23: 15: 16$ & $+18: 57: 40$ & 34.2 & $\mathrm{CV}$ & 8.91 & 255 & 7.90 & 5.41 & \\
\hline
\end{tabular}


Table 4

(Continued)

\begin{tabular}{lccccccccc}
\hline \hline Name & $\begin{array}{c}\text { R.A. }^{\mathrm{a}} \\
(\mathrm{J} 2000)\end{array}$ & Decl. & $\begin{array}{c}m-M^{\mathrm{b}} \\
(\mathrm{mag})\end{array}$ & Method $^{\mathrm{c}}$ & $\begin{array}{c}K^{\mathrm{d}} \\
(2 \mathrm{MASS})\end{array}$ & $\begin{array}{c}\sigma^{\mathrm{e}} \\
\left(\mathrm{km} \mathrm{s}^{-1}\right)\end{array}$ & $\begin{array}{r}M(\mathrm{BH})_{K} \mathrm{f}^{\mathrm{f}} \\
\left(10^{8} M_{\odot}\right)\end{array}$ & $\begin{array}{r}M_{(\mathrm{BH})_{\sigma}{ }^{\mathrm{g}}} \\
\left(10^{8} M_{\odot}\right)\end{array}$ & Notes $^{\mathrm{h}}$ \\
\hline NGC 7562 & $23: 15: 57$ & $+06: 41: 14$ & 33.8 & SBF & 8.32 & 256 & 9.03 & 5.51 \\
NGC 7600 & $23: 18: 53$ & $-07: 34: 49$ & 33.4 & CV & 8.91 & 210 & 3.56 & 2.10 \\
NGC 7618 & $23: 19: 47$ & $+42: 51: 09$ & 34.3 & CV & 9.04 & 298 & 7.35 & 11.53 \\
NGC 7626 & $23: 20: 42$ & $+08: 13: 01$ & 33.7 & SBF & 8.03 & 271 & 10.66 & 7.27 \\
NGC 7711 & $23: 35: 39$ & $+15: 18: 07$ & 33.8 & CV & 8.91 & 180 & 4.93 & 0.99 \\
NGC 7743 & $23: 44: 21$ & $+09: 56: 02$ & 31.6 & SBF & 8.42 & 84 & 0.97 & 0.02 \\
NGC 7785 & $23: 55: 19$ & $+05: 54: 57$ & 33.8 & $D_{n}-\sigma$ & 8.45 & 255 & 7.98 & 5.41 \\
\hline
\end{tabular}

Notes.

a J2000 coordinates, from the NASA/IPAC Extragalactic Database (NED, http://ned.ipac.caltech.edu).

${ }^{b}$ Distance modulus estimate, using the method identified in Column 5, as listed in the NASA/IPAC Extragalactic Database (NED, http://ned.ipac.caltech.edu).

${ }^{c}$ Method used in determining the distance modulus. For uniformity, preference was given to surface brightness fluctuation (SBF) distances, followed by Type Ia Supernova (SNIa) distances, distances based on the Tully-Fisher (TF) relation and $D_{n}-\sigma$ relations, and redshift distances (CV). The latter are calculated from the recessional velocity (as listed in NED) divided by $H_{0}=72 \mathrm{~km} \mathrm{~s}^{-1} \mathrm{Mpc}^{-1}$ (Freedman et al. 2000). Specific references can be found in NED.

d The total $K$-band magnitude derived from fit extrapolation, as listed in the 2MASS database (http://irsa.ipac.caltech. edu/cgi-bin/Gator/nph-dd, Jarrett 2000).

${ }^{\mathrm{e}}$ Average value of the stellar velocity dispersion, computed from all available sources following the precepts of Prugniel \& Simien (1996), as listed in the HyperLeda database (http://leda.univ-lyon1.fr/).

${ }^{\mathrm{f}}$ Black hole mass estimated from the $K$-band magnitude listed in Column 6, following Equation (7) of Vika et al. (2012).

g Black hole mass estimated from the stellar velocity dispersion listed in Column 7, following Equation (20) of Ferrarese \& Ford (2005).

${ }^{\mathrm{h}}$ Black hole masses based on dynamical models applied to spatially resolved kinematics are listed when available.

References. (1) Sarzi et al. 2001; (2) van der Marel \& van den Bosch 1998; (3) Gebhardt et al. 2007; (4) Barth et al. 2001; (5) Gultekin et al. 2009; (6) Ferrarese \& Ford 1999.

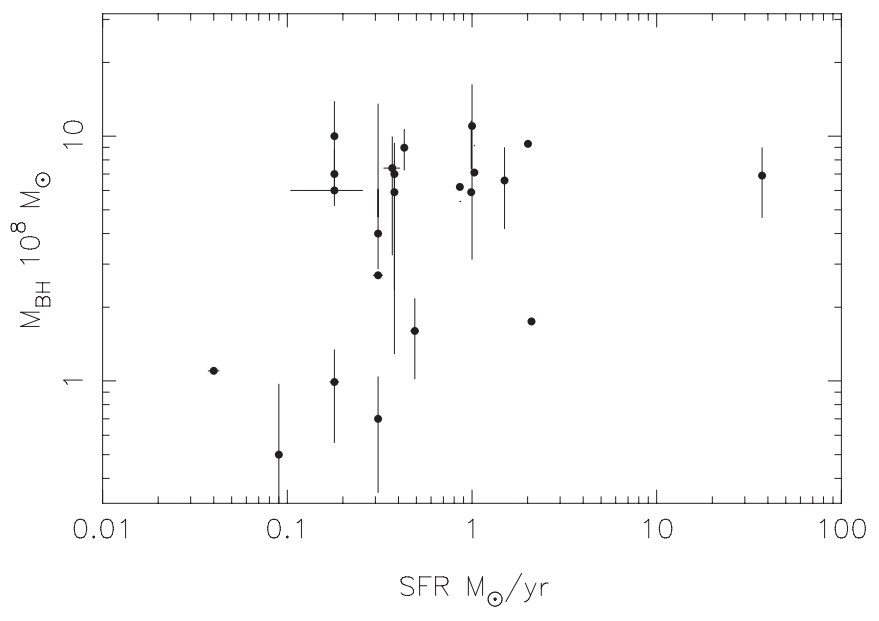

Figure 9. SFR and black hole mass from Tables 2 and 4.

Photoionization by hard radiation from close to the $\mathrm{BH}$ could also strengthen $\mathrm{Br} \gamma$ over the value due to star formation.

\section{CONCLUSIONS}

Some $20 \%$ of ellipticals in the Brown et al. (2011) sample have emission-line nuclei, which will be followed up with IFU spectrographs on AO-equipped telescopes. The complete sample may have a somewhat lower emission fraction, as galaxies with optical emission lines (where this was known) tended to be observed first in order to provide immediate targets for IFU observing. Emission-line nuclei are accompanied by more powerful radio sources at a given galaxy mass.

The SFR in these nuclei has a median value of $0.4 M_{\odot} \mathrm{yr}^{-1}$ and must be intermittent to attain the median $\mathrm{BH}$ mass of $5 \times 10^{8} M_{\odot}$ of these galaxies.

Thanks go to Eilat Glickman for sharing her observing expertise at the Palomar 200 inch telescope and her copy of the Triplespec Spextool IDL program. This paper has also made use of IRAF, which is distributed by NOAO. NOAO is operated by AURA under a cooperative agreement with NSF. The Caltech-Swinburne Collaborative Agreement allows the first author access to the Hale and Keck telescopes. Survey astronomy is supported by the Australian Research Council through CAASTRO. ${ }^{14}$ The Centre for All-sky Astrophysics is an Australian Research Council Centre of Excellence, funded by grant CE11001020. G.C. acknowledges support from STFC grants ST/H002456/1 and ST/I003673/1. M.B. acknowledges the support from the Australian Research Council via Future Fellowship FT100100280 and Discovery Project DP110102174. We thank an anonymous referee for comments which improved the paper.

\section{REFERENCES}

Antonucci, R., Hickson, P., Miller, J. S., \& Olszewski, E. W. 1987, AJ, 93, 785

Balzano, V. 1983, ApJ, 268, 602

Barth, A., Greene, J., \& Ho, L. 2005, ApJ, 619, L151

14 www.caastro.org 
Barth, A., Sarzi, M., Rix, H.-W., et al. 2001, ApJ, 555, 685

Best, P., Kaiser, C., Heckman, T., \& Kauffmann, G. 2006, MNRAS, 368, L67

Bower, R., Benson, A., Malbon, R., et al. 2006, MNRAS, 370, 645

Brown, M. J. I., Jannuzi, B., Floyd, D., \& Mould, J. 2011, ApJ, 731, 41

Cesetti, M., Ivanov, V. D., Morelli, L., et al. 2009, A\&A, 497, 41

Cushing, M., Vacca, W., \& Rayner, J. 2004, PASP, 116, 362

Fabbiano, G., Gioia, I. M., \& Trinchieri, G. 1989, ApJ, 347, 127

Ferrarese, L., \& Ford, H. F. 1999, ApJ, 515, 583

Ferrarese, L., \& Ford, H. F. 2005, Space Sci. Rev., 116, 523

Ferrarese, L., \& Merritt, D. 2000, ApJ, 539, L9

Freedman, W. L., Madore, B. F., Gibson, B. K., et al. 2000, ApJ, 553, 47

Gebhardt, K., Bender, R., Bower, G., et al. 2000, ApJ, 539, L13

Gebhardt, K., Lauer, T. R., Pinkney, J., et al. 2007, ApJ, 671, 1321

Graham, A. 2007, MNRAS, 379, 711

Granato, G. L., De Zotti, G., Silva, L., Bressan, A., \& Danese, L. 2004, ApJ, 600,580

Greene, J. E., \& Ho, L. C. 2006, ApJ, 641, L21

Greene, J. E., \& Ho, L. C. 2007, ApJ, 667, 131

Gultekin, K., Richstone, D. O., Gebhardt, K., et al. 2009, ApJ, 695, 1577

Hardcastle, M., Evans, D., \& Croston, J. 2006, MNRAS, 376, 1849

Ho, L. 1999, ApJ, 510, 631

Ho, L. 2008, ARA\&A, 46, 475

Hopkins, P. F., \& Hernquist, L. 2006, ApJS, 166, 1

Hopkins, P. F., Hernquist, L., Cox, T. J., \& Kereš, D. 2008, ApJS, 175, 356
Jahnke, K., \& Maccio, A. 2010, ApJ, 734, 92

Jarrett, T. H. 2000, PASP, 112, 1008

Kennicutt, R. 1998, ARA\&A, 36, 189

Kormendy, J. 1993, in The Nearest Active Galaxies, ed. J. Beckman, L. Colina, \& H. Netzer (Madrid: Consejo Superior de Investigaciones Cientificas), 197

Kormendy, J. 2004, Coevolution of Black Holes and Galaxies (Carnegie Observatories Astrophysics Series, Vol. 1; Cambridge: Cambridge Univ. Press)

Kormendy, J., \& Richstone, D. O. 1995, ARA\&A, 33, 581

Lynden-Bell, D., \& Rees, M. 1971, MNRAS, 152, 461

Magorrian, J., Tremaine, S., Richstone, D., et al. 1998, AJ, 115, 2285

Mayes, A. J., Evans, A., \& Pearce, G. 1985, A\&A, 143, 327

Murakami, H., Baba, H., Barthel, P., et al. 2007, PASJ, 59, 369

Prugniel, P., \& Simien, F. 1996, A\&A, 309, 749

Riffel, R., Rodrguez-Ardila, A., \& Pastoriza, M. G. 2006, A\&A, 457, 61

Sadler, E. M., Jenkins, C. R., \& Kotanyi, C. G. 1989, MNRAS, 240, 591

Slee, O. B., Sadler, E. M., Reynolds, J. E., \& Ekers, R. D. 1994, MNRAS, 269, 928

Sarzi, M., Rix, H.-W., Shields, J. C., et al. 2001, ApJ, 550, 65

Springel, V., Di Matteo, T., \& Hernquist, L. 2005, MNRAS, 361, 776

van der Marel, R. P., \& van den Bosch, F. C. 1998, AJ, 116, 2220

Veron-Cetty, M., \& Veron, P. 2006, A\&A, 455, 773

Vika, M., Driver, S. P., Cameron, E., Kelvin, L., \& Robotham, A. 2012, MNRAS, 419, 2264 